\title{
Veranstaltungen der AOTrauma auf dem DKOU
}

$\square$ Karl-Heinz Frosch, Philip Wilbrandt

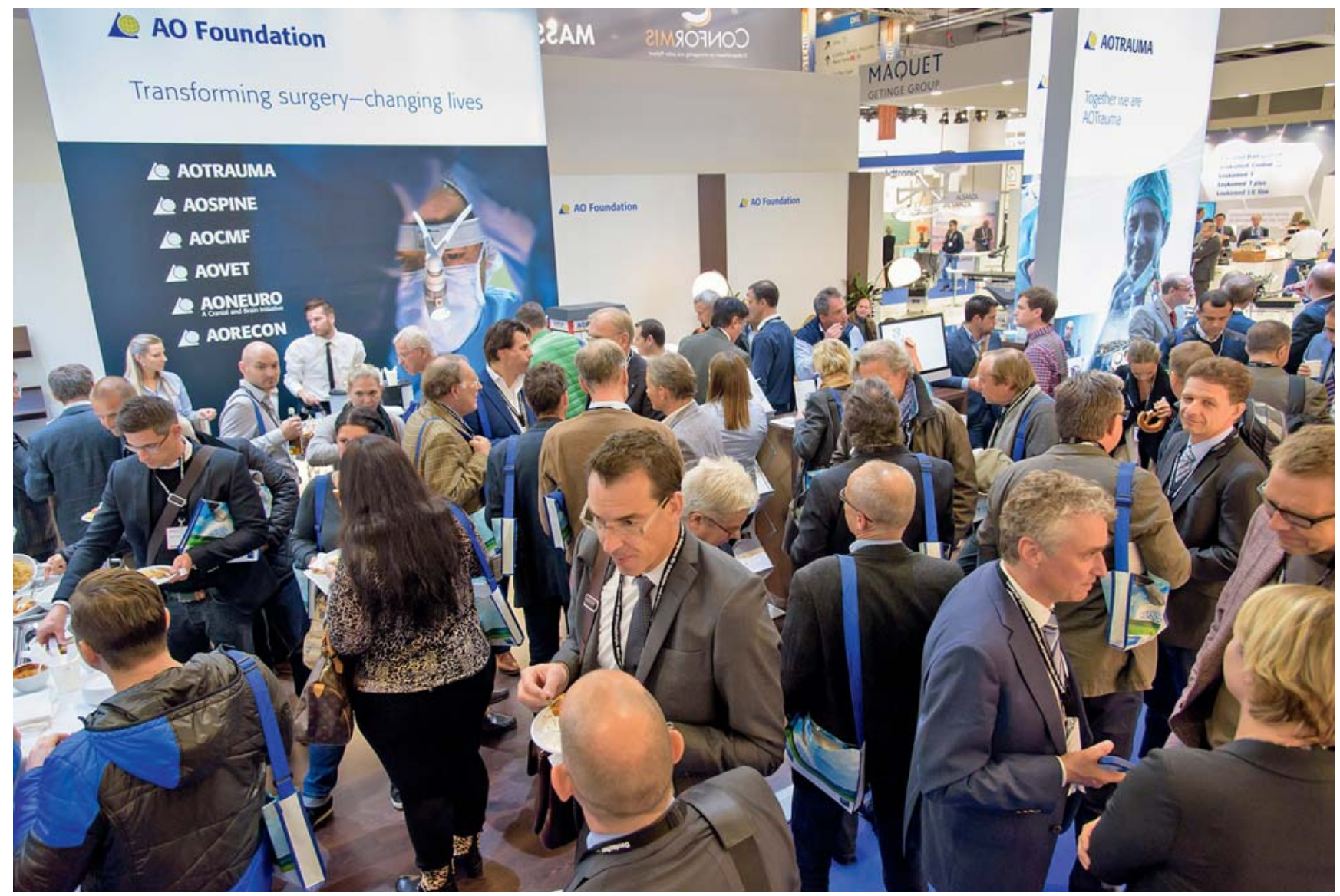

Abb. 1 Get-together am AO-Stand auf dem DKOU 2015. Schwarz, Starface (mit freundlicher Genehmigung der AO Foundation).

Auf dem DKOU 2016 richtet die AO diverse wissenschaftliche Sitzungen, Satelliten-Symposien und Informationsveranstaltungen am AO-Stand sowie 3 Gettogether-Empfänge nach ausgewählten
AO-Veranstaltungen aus. Zum ersten Mal finden eine Sitzung der Akademie und die Auswahlsitzung für die Nachwuchsförderung der AOTrauma Deutschland statt.
Karl-Heinz Frosch

Hamburg

\section{Philip Wilbrandt}

Rechlin 
Tab. 1 Veranstaltungen der AO Stiftung auf dem DKOU 2016

\begin{tabular}{|c|c|c|c|c|c|}
\hline Datum & Uhrzeit & $\begin{array}{l}\text { DKOU } \\
\text { Programm } \\
\text { Nummer }\end{array}$ & Raum & Thema der Veranstaltung & Vorsitz \\
\hline $\begin{array}{l}\text { Dienstag, } \\
\text { 25. Oktober } \\
2016\end{array}$ & $\begin{array}{l}14: 30- \\
16: 00\end{array}$ & IN13 & New York 1 & $\begin{array}{l}\text { AO Research Institute, ORS: Acute cartilage } \\
\text { injury }\end{array}$ & R. Brenner, N. Südkamp \\
\hline \multirow{12}{*}{$\begin{array}{l}\text { Mittwoch, } \\
\text { 26. Oktober } \\
2016\end{array}$} & $\begin{array}{l}\text { 09:00- } \\
10: 30\end{array}$ & ER11 & Helsinki & $\begin{array}{l}\text { AOSpine Symposium: Alterstraumatologie der } \\
\text { Wirbelsäule - Komplikationsmanagement }\end{array}$ & O. Gonschorek, M. Schultheiß \\
\hline & $\begin{array}{l}09: 00- \\
10: 30\end{array}$ & IN18 & New York 1 & $\begin{array}{l}\text { AOTrauma Symposium: Non-weight bearing } \\
\text { after fracture care: where is the evidence? }\end{array}$ & F. Bonnaire, H. Winker \\
\hline & $\begin{array}{l}10: 30- \\
11: 15\end{array}$ & Empfang & Stand AO-Stiftung & $\begin{array}{l}\text { AOTrauma Europa: Empfang/Umtrunk am } \\
\text { Stand der AO-Stiftung }\end{array}$ & \\
\hline & $\begin{array}{l}10: 30- \\
11: 30\end{array}$ & & Stand AO-Stiftung & AO AutoGauge: Smart Drilling Practical & \\
\hline & $\begin{array}{l}11.00- \\
12.30\end{array}$ & IN19 & New York 1 & AO Research Institute: Smart Surgery & N. Südkamp \\
\hline & $\begin{array}{l}11.00- \\
12.30\end{array}$ & TI12 & Festsaal & $\begin{array}{l}\text { AOT Deutschland „Zurück in die Zukunft: } \\
\text { Renaissance vergessener Verfahren“, inkl. } \\
\text { Vergabe Wissenschaftspreis } 2016\end{array}$ & R. Babst, M. Raschke \\
\hline & $\begin{array}{l}13: 00- \\
14: 00\end{array}$ & & Stand AO-Stiftung & Smart Implants: AO Fracture Monitor Session & M. Windolf \\
\hline & $\begin{array}{l}14.30- \\
16.00\end{array}$ & IN20 & New York 1 & AOTrauma Symposium: Augmented Implants & M. Gosch, R. Simmermacher \\
\hline & $\begin{array}{l}15: 00- \\
16: 00\end{array}$ & & Stand AO-Stiftung & AO AutoGauge: Smart Drilling Practical & \\
\hline & $\begin{array}{l}16: 00- \\
16: 30\end{array}$ & Empfang & Stand AO-Stiftung & $\begin{array}{l}\text { AOTrauma Europa: Empfang/Umtrunk am } \\
\text { Stand der AO-Stiftung }\end{array}$ & \\
\hline & $\begin{array}{l}16.30- \\
18.00\end{array}$ & IN 21 & New York 1 & $\begin{array}{l}\text { AOCMF Symposium: Midface trauma - } \\
\text { who cares? }\end{array}$ & N. Futran, E. Kollig \\
\hline & $\begin{array}{l}16.30- \\
18.00\end{array}$ & FG14 & London 3 & AOT D-A-CH: Implantatinfektionen & T. Neubauer, M. Raschke \\
\hline \multirow[t]{6}{*}{$\begin{array}{l}\text { Donnerstag, } \\
27 . \text { Oktober } \\
2016\end{array}$} & $\begin{array}{l}09.00- \\
10.30\end{array}$ & IN26 & New York 1 & $\begin{array}{l}\text { AOTrauma/AORecon Symposium: Prevention } \\
\text { and management of periprosthetic fractures - } \\
\text { part 1: knee }\end{array}$ & S. Bavonratanavech, J. Wilber \\
\hline & $\begin{array}{l}10: 00- \\
11: 00\end{array}$ & & AO Stiftung Stand & AO AutoGauge: Smart Drilling Practical & \\
\hline & $\begin{array}{l}11.00- \\
12.30\end{array}$ & IN27 & New York 1 & $\begin{array}{l}\text { AOSpine Symposium: Controversies in spinal } \\
\text { surgery: is therapy in Germany too aggressive? }\end{array}$ & L. Benneker \\
\hline & $\begin{array}{l}12: 30- \\
13: 30\end{array}$ & Empfang & AO Stiftung Stand & $\begin{array}{l}\text { AOTrauma Deutschland: Empfang/Umtrunk am } \\
\text { Stand der AO-Stiftung }\end{array}$ & \\
\hline & $\begin{array}{l}13: 30- \\
14: 30\end{array}$ & & AO Stiftung Stand & Smart Implants: AO Fracture Monitor Session & M. Windolf \\
\hline & $\begin{array}{l}14.30- \\
16.00\end{array}$ & IN 28 & New York 1 & $\begin{array}{l}\text { AOTrauma/AORecon Symposium: Prevention } \\
\text { and management of periprosthetic fractures - } \\
\text { part 2: hip }\end{array}$ & S. Bavonratanavech, C. Perka \\
\hline \multirow{2}{*}{$\begin{array}{l}\text { Freitag, } \\
\text { 28. Oktober } \\
2016\end{array}$} & $\begin{array}{l}09.00- \\
10.30\end{array}$ & VE54 & Weimar 1 & $\begin{array}{l}\text { AOT Deutschland: Auswahlsitzung Nachwuchs- } \\
\text { förderung }\end{array}$ & G. Duda, H. Pape, R. Stange \\
\hline & $\begin{array}{l}14.30- \\
16.00\end{array}$ & VE78 & 411 & $\begin{array}{l}\text { AOT Deutschland, Akademie: Wohin entwickelt } \\
\text { sich die Unfallchirurgie? }\end{array}$ & W. Böcker, H. Oestern \\
\hline
\end{tabular}

